\title{
Organizational Pressure Dimensions Measuring of "Post-80s" Employees and Its Implications for Talent Strategy
}

\author{
Dongjin Yang, Li Yang \\ Management School, Jinan University, Guangzhou, China \\ Email: 512612437@qq.com
}

Received 21 October 2014; revised 21 November 2014; accepted 27 November 2014

Copyright (C) 2014 by authors and Scientific Research Publishing Inc.

This work is licensed under the Creative Commons Attribution International License (CC BY). http://creativecommons.org/licenses/by/4.0/

(c) (i) Open Access

\begin{abstract}
This article adopts empirical research methods to measure the content of organizational pressure about "post-80s" employees. Exploratory factor analysis and confirmatory factor analysis are used to extract and verify 6 factors of organizational pressure. The 6 factors are workload, monotonous work, role conflict, interpersonal relationships, career development and organizational system. Based on the 6 dimensions of organization pressure, we propose some measures the organization should take, providing references for corporate human resources management.
\end{abstract}

\section{Keywords}

“Post-80s", Organizational Pressure, Measures

\section{Issue Raised}

Since the reform and opening up, China has begun to slowly start the prelude of social transformation. The traditional Chinese social structure is divided, and the most prominent conflicts are the culture and values. Chinese economy has developed fast during this period, but accompanied by various contradictions and shocks. The fact is that many corporate employees are faced with a strong sense of crisis and stress in such a competitive environment [1]. Chinese Academy of Sciences Institute of Psychology released the latest survey results on the pressure of people: during the social transformation, the people aged 20 - 30 years old are faced with the most pressure, which are just "post-80s" [2]. On May 26, 2006, the death of 25-year-old Huawei staff, Hu Xinyu, becomes China's first "karoshi" event, which was also the 6th unnatural death event of Huawei since 2008. In 2010, there were 12 suicides by jump down from upstairs in Foxconn, which caused 10 dead and 2 injured. The suicides are young people aged 18 - 29. In 2011, the "karoshi" of 25-year-old female postgraduate in $\mathrm{PwC}$ is 
equally unfortunate. These events reflect that the psychological status of our employees is not optimistic, and the pressure of the staff within the organization can't be ignored.

There is a special group that draws our attention in these events. They are "post-80s" employees of enterprises. Why do they often become victims of stress? What aspects of the organization actually bring heavy pressure for employees? These are worth pondering of society and organizational managers. We also noted that work environments of "post-80s" have different characteristics in contrast with previous, and the problems they face also have characteristics of the times. The political, economic and cultural growth background of "post-80s" has a clear distinction with the " $60 \mathrm{~s}$ " and "70s". First, they are growing in an open environment, widely influenced by western culture and lifestyles; second, they are growing in the times of market economy, living in an atmosphere of competition and profit; third, the continuous improvement of living standards makes them experience less life adversities and setbacks; fourth, part of them are the only child in their family, and non-only children also are growing in the low birth rate families, enjoying much caring of parents and grandparents; fifth, they are the generation accompanied by the growth of the Internet, which makes them get rich information quickly and enjoy a wide range of social interaction channels and open space for self-expression. All of those are what their predecessors have not ever experienced. These social factors construct their distinctive characteristics, so that they become very unique generation in modern Chinese history [3]. Yu Shuanghao did an interview on "post-80s". She proposed the opinion that the environment of reforming and opening created a unique "post-80s" generation. They have unique personality, psychological characteristics and lifestyle [4]. They are also suffering unique mental pressure and mental confusion. Yi Xinfa also thought that the psychological formation of "post- 80 s" was the result of multifaceted impact on society, family, education and personal awareness [5]. Guo Jing studied the living conditions of "post-80s". He found that they had 6 characteristics which were high degree, high consumption, high emotional intelligence, high expectations, high CQ and high sense of innovation [6]. The article analyzed their state of mind and psychological. Wu Yue elaborated the work and economic pressures of "post-80s" from a psychological perspective [7].

Enterprise paying attention to and managing employee stress can fully reflect the people-oriented management methods, which are conducive to building a good corporate culture and enhancing employee loyalty to the enterprise. Effective stress management is not only a prerequisite of developing healthy, happy and efficient staff, but also the key for enterprise to attract and retain qualified personnel (Zhang, 2006) [8]. So, how to conduct effective stress management plays an important role on sustainable development of enterprise. Stress management also becomes the focus of enterprise management. Today, the "post-80s", these new generation employees, have been working, and gradually become the backbone of the organization. Whether the pressure they are facing can be effectively addressed is related to not only the staff but also the future development of the organization. This paper will study the empirical data and find out what factors organization pressure source of "post-80s" employees contains?

\section{A Study Design and the Main Concepts}

\subsection{Study Design}

Through questionnaire on the sample, we obtain the data on the organization stress source of "post- 80 s" employees, and measure and determine the content structure of organizational stress by use of exploratory factor analysis and confirmatory factor analysis, then build an objective organization pressure evaluation system. At last, we discuss the talent strategy for organization to retain "post-80s" new employees.

\subsection{Key Concepts}

(1) Organizational pressure. In terms of the definition of pressure, different scholars study give a different definition based on different study directions. Looking at the definition of stress researchers made, the definition can be summarized mainly in three directions as follows: irritation doctrine (stimulus definition), reaction theory (response definition) and the interaction theory (relational definition) [9]. The biggest difference between organizational pressure and stress is that the study of organizational pressure is the microscopic study of organizational stress problems. Although Lazarus pressure interaction theory is more complete and comprehensive, it takes individual differences into account and is often accepted by most scholars. But although there are differences inside the group of "post-80s", they certainly have common characteristics growing up in the same politi- 
cal, economic, and cultural background. This paper is to study their different organizational pressure caused by the common characteristics. Thus we are in favor of stimulus definition and propose that pressure refers to those external events which cause the individual anxiety, restlessness and irritation. Organization pressure refers to the external factors which cause staff anxiety and restlessness.

(2) "Post-80s". They are mainly two definitions on the "post-80s". One is narrow definition, which refers to people born in 1980-1989. Another is board definition, which refers to the people born after 1980. This article study board "post-80s". We quote the definition by Xia, who is the sociology professor at Peking University. "Post-80s" refers to those born after 1980, and growing up in a better material environment with the background of reforming and opening up [10].

\section{Dimensions Measurement of Organizational Pressure}

\subsection{Pre-Trial Preparation and Survey Questionnaire}

A semi-open questionnaire made according to expert interviews and the existing literature relevant to content of organization pressure, to collect comprehensive content elements of organizational pressure. A total of 55 questionnaires are handed out, of which 30 were electronic questionnaires, 25 were paper questionnaires. At last, 50 organizational pressure elements were collected. Then we invited the relevant experts and scholars to review each term to modify and eliminate duplication ambiguous terms. Eventually the organization pressure questionnaire with 45 terms was formed, which contained work load, monotonous work, employee relations, corporate culture, role ambiguity, role conflict, wages and benefits, organizational systems, career development. Pre-test questionnaires were distributed 260 copies and 234 copies were returned. There are a total of 225 valid questionnaires finally after giving up the questionnaires with missing values. SPSS is used to do factor analysis and eight major interpretable factors are extracted. But part of the factors were with low factor loadings, and scree plot indicates nine factors existing, so pre-test questionnaire result is not ideal. The formal questionnaire was formed after deleting and modifying the terms with lower descriptive analysis score and lower factor loadings in factor analysis.

\subsection{Exploratory Factor Analysis}

432 formal questionnaires were distributed and 412 valid questionnaires are returned, of which 202 copies were used for exploratory factor analysis, and the remaining 210 questionnaires were used for confirmatory factor analysis. First, we do descriptive analysis, deleting the items scored 3 or less. Principal component analysis included factor analysis, the KMO, Bartlett's test and degree of freedom and so on. We did several principal component analyses on the remaining items. Finally 6 factors of organizational pressure were formed and every factor contain 3 terms. The cumulative variance contribution rate is amounted to $74.06 \%$ in Table 1 . In the last principal component analysis, KMO value is 0.740 . The result of Bartlett's test is 1568.837 . Degree of freedom is 153 , which is significant. All results indicated that this sample was suitable for factor analysis.

\subsection{Factor Named}

The content and factor loadings of each factor are shown in Table 2. From the table, we know that the content of the factors are consistent with what we have considered.

Workload, employees undertake too much work, often working overtime. In addition, they complain about the tedious work.

Monotonic work, if the work content of employees is always consistent, the employees will get bored of the work.

Role conflict, employees often receive contradictory requests from two or more different superiors. In addition, the employees need to complete other work except their own responsibility.

Interpersonal relationships, the employees think they cannot get the support form superiors, and cannot get along well with their workmates.

Career development, employees propose that they are not likely to get promoted in their company and can't new knowledge and skills.

Organizational system, employees think they are not paid fairly and there is not enough performance feedback. 
Table 1. Principal component analysisofexploratory factor analysis $(\mathrm{N}=202)$.

\begin{tabular}{ccccccc}
\hline Principal component & 1 & 2 & 3 & 4 & 5 & 6 \\
\hline Variance contribution rate $\%$ & 13.85 & 13.2 & 12.97 & 12.28 & 11.65 & 10.11 \\
The cumulative variance contribution rate $\%$ & 13.85 & 27.05 & 40.01 & 52.3 & 63.95 & 74.06 \\
\hline
\end{tabular}

Table 2. Content and factor loadings of each factor $(\mathrm{N}=202)$.

\begin{tabular}{|c|c|c|c|c|c|}
\hline $\mathrm{F} 1$ & $\mathrm{~F} 2$ & F3 & $\mathrm{F} 4$ & F5 & F6 \\
\hline Y1 0.830 & Y7 0.814 & Y14 0.862 & Y19 0.734 & Y28 0.844 & Y33 0.822 \\
\hline Y5 0.823 & Y8 0.853 & Y15 0.835 & Y22 0.738 & Y30 0.842 & Y360.877 \\
\hline Y6 0.786 & Y9 0.879 & Y16 0.771 & Y24 0.821 & Y31 0.843 & Y37 0.862 \\
\hline
\end{tabular}

F1: Workload; Y1: Overtime work; Y5: Workload; Y6: Tedious work; F2: Monotonic work; Y7: Boring Working; Y8: No challenge; Y9: Responsibilities unchanged; F3: Role conflict; Y14: Conflicting demands; Y15: Non-own work; Y16: Reporting unknown; F4: Interpersonal Relationships; Y19: Superior support; Y22: Collaboration with colleagues; Y24: Personality conflicts; F5: Career development; Y28: Promotion; Y30: Career goal; Y31: New knowledge and skills; F6: Organizational system; Y33: Emolument management; Y36: Performance feedback; Y37: Complex hierarchy.

\subsection{Confirmatory Factor Analysis}

The scale or questionnaire structure validity is established through exploratory factor analysis. Confirmatory factor analysis (CFA) can be used to test its relevance and authenticity. We use structural equation model to do confirmatory factor analysis. Considering the space, this article discusses only the 4 better-fitting models. The results of 4 models are showed in Table 3. Comparing the fit Indexes of four kinds of assumption models, we will find that Model 2 (revised Model 1) is the most ideal assumption model, which is the best hypothetical model. The above analysis shows that the results of exploratory factor analysis has been verified, indicating that the organizational pressure has a stable structure with 6 dimensions.

\subsection{Reliability and Validity}

\subsubsection{Reliability Test}

Using homogeneous reliability (Cronbach $\alpha$ coefficient) to test the reliability of the questionnaire commitment. Factor levels for each subscale and total scale internal consistency test results are shown in Table 4. F1, F2, F3, F5, $\alpha$ coefficient F6 subscales above 0.70 , indicating that F1, F2, F3, F5, F6 subscale reliability of measurement results is quite good $\alpha$ coefficient F4 subscales above 0.50 , indicating that F5 subscale reliability of measurement results can be accepted. The overall questionnaire coefficient was 0.782 , indicating the reliability of measurement results is quite good.

\subsubsection{Validity}

(1) Construct validity. The main purpose of this study is to investigate the content structure of organizational pressure. factor analysis is the most commonly used method to examine the construct validity of the questionnaire, the results in Table 1 indicate that the organizational pressure structure is very clearly organized by 6 factors, which amounted to $74.06 \%$ of the total variance explained. Construct validity of the questionnaire was good. In addition, we selected 210 samples to do confirmatory factor analysis on 6-factor model. The indicators show that 6 -factor model is the best model of organizational stress, and further validate the construct validity of organizational pressure is ideal.

(2) Content validity. We can evaluate the content validity by comparing the measure results of a study with the results of existing research. Robbins considers organizational factors of employee stress contain mission requirements, the role demands, interpersonal requirements, organizational structure, organization and leadership [11]. $\mathrm{Xu}$ is one of the domestic research scholars studying work force pressure, $\mathrm{Xu}$ argues source of job stress can be roughly divided into two categories: one is from the work itself, which is called inside work stress. It contains the work content, work standards and other factors; the other is out of work itself, which is called outside work stress. It contains the working environment, relationships and other factors [12]. After evaluation and comparison, the content measured has good content validity and is suitable for the employees similar to the sample. 
Table 3. Main fit index assumptions of the model $(\mathrm{N}=210)$.

\begin{tabular}{|c|c|c|c|c|c|c|c|c|}
\hline \multirow{2}{*}{$\begin{array}{l}\text { Hypothesis } \\
\text { model }\end{array}$} & \multicolumn{3}{|c|}{ Absolute fit index } & \multicolumn{2}{|c|}{ Relative fit index } & \multicolumn{3}{|c|}{ Alternative index } \\
\hline & CMIN (p) & $\mathrm{DF}$ & $\mathrm{CMIN} / \mathrm{DF}$ & NFI & PNFI & NCP & RMSEA & CFI \\
\hline Model 1 & $103.118(0.035)$ & 83 & 1.256 & 0.901 & 0.618 & 22.213 & 0.021 & 0.921 \\
\hline Model 2 & $87.321(0.068)$ & 81 & 1.357 & 0.902 & 0.673 & 17.427 & 0.018 & 0.932 \\
\hline Model 3 & $101.625(0.071)$ & 85 & 1.263 & 0.823 & 0.702 & 26.223 & 0.031 & 0.931 \\
\hline Model 4 & $111.638(0.031)$ & 84 & 1.310 & 0.8881 & 0.711 & 25.368 & 0.029 & 0.985 \\
\hline Optimal value & Low better $(>0.05)$ & - & $<2$ & $>0.9$ & $>0.5$ & Low better & $<0.1$ & $>0.9$ \\
\hline
\end{tabular}

Table 4. Results of reliability and validity test $(\mathrm{N}=210)$.

\begin{tabular}{ccccccccc} 
Scale & F1 & F2 & F3 & F4 & F5 & F6 & Total Table \\
\hline$\alpha$ & 0.87 & 0.869 & 0.765 & 0.503 & 0.822 & 0.833 & 0.782 \\
\hline
\end{tabular}

\section{Talent Strategies according to the 6 Dimensions}

According to the analysis of the foregoing, it can be seen that the 6 dimensions of organizational stress model proposed by exploratory factor analysis has been verified by structural equation model. Talent strategies according to the 6 dimensions are as follows:

(1) Workloads, through the interview with "post-80s", we can know that "post-80s" are suffering heavy workload. They complain that they always have too much to do, and they often need work over time to complete their work. The HR of the organization should evaluate whether the company is in need of more employees to make the organization work well. We cannot allow an employee to complete the assignments of two or more employees. In addition, we should make employees work overtime as less as possible. The HR need to confirm whether the efficiency of the employees is too low. If possible, the employees should often obtain new skills to improve efficiency.

(2) Working monotonous, employees will be tired of the monotonous and repetitive work, particularly the staff whose job responsibility has been the same for a long time. The organization can take measures to change the status of the staff. As we know, new things can always arouse people interests. The organization can provide rotation for the staff, so the staff can choose to transfer to another position with different responsibilities. In addition, we can provide the employees the opportunities to obtain new knowledge and skills, so that he is able to choose to apply for another position that they are interested in. What's more, we can provide these staff rotation possibilities.HR can arrange staff training to learn new knowledge and skills so that employees have the ability and skills to match other jobs. In addition, we can provide recreational facilities in the company, making employees' work life not that monotonous.

(3) Career development, the most important thing is that we need make a clear career development plan for our staff. With the era of knowledge economy coming, companies have higher quality demand for its employees. Most of the "post-80s" employees feel necessary to update their knowledge to keep up with the speed of technology upgrade, especially the technical staff and the Business staff. Therefore, the organization should strengthen job skills of employees, providing training to meet demand of the staff. If a company is willing to take responsibility of staff career planning, combining the employee career development and the development of enterprises closely, it can not only relieve the staff pressure, but also increase the loyalty of employee to the organization, forming a win-win situation.

(4) Personnel relationship, pleasant atmosphere in the organization enables employees to work in a relaxed mood, and help improve job performance. Team is more and more important in modern management, so we can't ignore the atmosphere and relationship in workplace. Coupled with modern management with particular emphasis on the importance of teamwork, interpersonal skills can't be ignored.

(5) Role conflict, many staffs suffer from the pressure of role conflict, especially frontline staffs and senior level managers. The organization can provide training, communication or appropriate and accurate information on the role of staff analysis, and clarify job roles, role expectations, the obligations and responsibilities in the organization, so that employees can accurately understand, recognize the work expectations, needs, clear objectives. In this way, we can reduce the pressure generated by role conflict. When working structure and staffing 
company system changes, we can make use of role analysis to eliminate stress. When there is a conflict of roles in the organization, you can use the role negotiations to resolve.

(6) Organization system, the pressure of organizational system which employees suffer from is mainly the pressure of payment system. All we know is that payment is closely relevant with the performance of the employees. What we need to do is to improve the performance appraisal system. Indicators should be set realistic, reasonable. In addition, the organization should provide feedback on staff performance. When the staff has outstanding performance, they should be rewarded. When the staff has poor performance, they should be warned or punished. In this way, it will enhance the sense of fairness to the employees.

\section{Research Limitations and Innovation}

The main limitation of the study is the representativeness of the sample. In this paper, mangers of management functions account for about half of the total selected sample. There are more than $70 \%$ the interviewed people coming from the enterprise with more than 1000 persons. The conclusions of this article may be not suitable for the senior managers and technical personnel.

The main innovations of this paper are in the following two aspects. First, we propose, measure and verify the structure dimensions of organizational pressure, which provide a relatively stable level indicator system for enterprise and employees to evaluate the level of organizational stress. Second, the research indicates that the content of organizational stress for "post-80s" is different from before, which embodies personality traits characteristics of "post-80s".

\section{References}

[1] Chen, Z.X. (2005) Principal Component Factor Structure Analysis of Knowledge Workers Working Pressure Source. Industrial Engineering and Management, 4, 26-30.

[2] Deng, F.D. (2011) Comparative Study Psychology of Enterprise "Post-70" and "Post-80" Staff. Ph.D. Thesis, Hunan University, Changsha.

[3] Li, C.L. (2013) Evolution of "Post-80" Phenomenon. Heilongjiang Social Sciences, 1, 82-87.

[4] Yu, S.H. (2009) "Post-80" in the Eyes of "Post-80". Management of Technology, 9, 9-14.

[5] Yi, X.F. (2006) Why Are Not Young People Dedicated. Science and Culture, 2, 31-33.

[6] Guo, J. (2008) Living Conditions and Countermeasures of Chinese "Post-80" Generation. Liaoning League, 5, 12-14.

[7] Wu, Y. (2010) "Post-80" Employee Stress Management from a Psychological Point of View. Chinese Businessmen, 7, $62-64$.

[8] Zhang, X.C. (2006) Employee Assistance Program: Theory and Practice of Chinese EAP. 5th Edition, China Social Science Press, Beijing.

[9] Mansoor, M., Fida, S., Nasir, S. and Ahmad, Z. (2011) The Impact of Job Stress on Employee Job Satisfaction: A Study on Telecommunication Sector of Pakistan. Journal of Business Studies Quarterly, 2, 50-56.

[10] Xiao, X.K. (2007) Stress Management Analysis of "Post-80" Employee. Human Resources Development, 4, 55-56.

[11] Robbins, S.P. (1992) Organizational Stress, Job Satisfaction and Job Performance: Where Do We Go From Here? Journal of Management, 2, 353-374.

[12] Xu, X.D. (2007) Working Pressure and Exogenous Pressures Structural Model of Endogenous Managers. Management Engineering, 1, 36-40. 
Scientific Research Publishing (SCIRP) is one of the largest Open Access journal publishers. It is currently publishing more than 200 open access, online, peer-reviewed journals covering a wide range of academic disciplines. SCIRP serves the worldwide academic communities and contributes to the progress and application of science with its publication.

Other selected journals from SCIRP are listed as below. Submit your manuscript to us via either submit@scirp.org or Online Submission Portal.
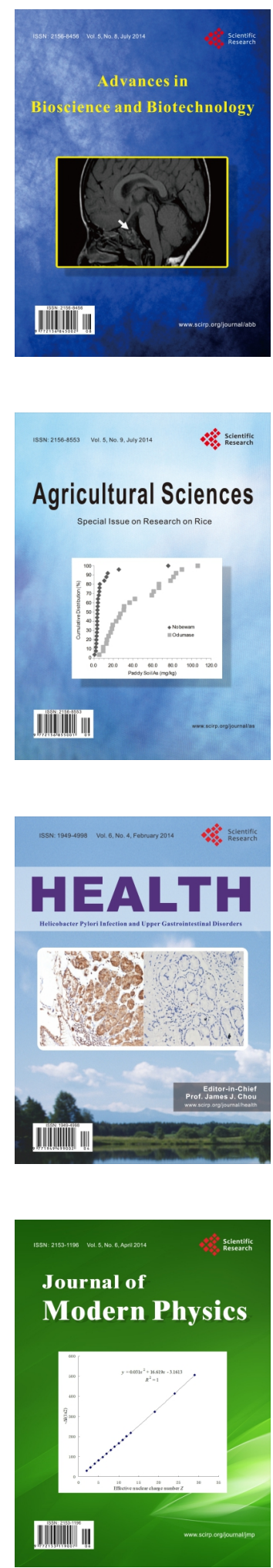
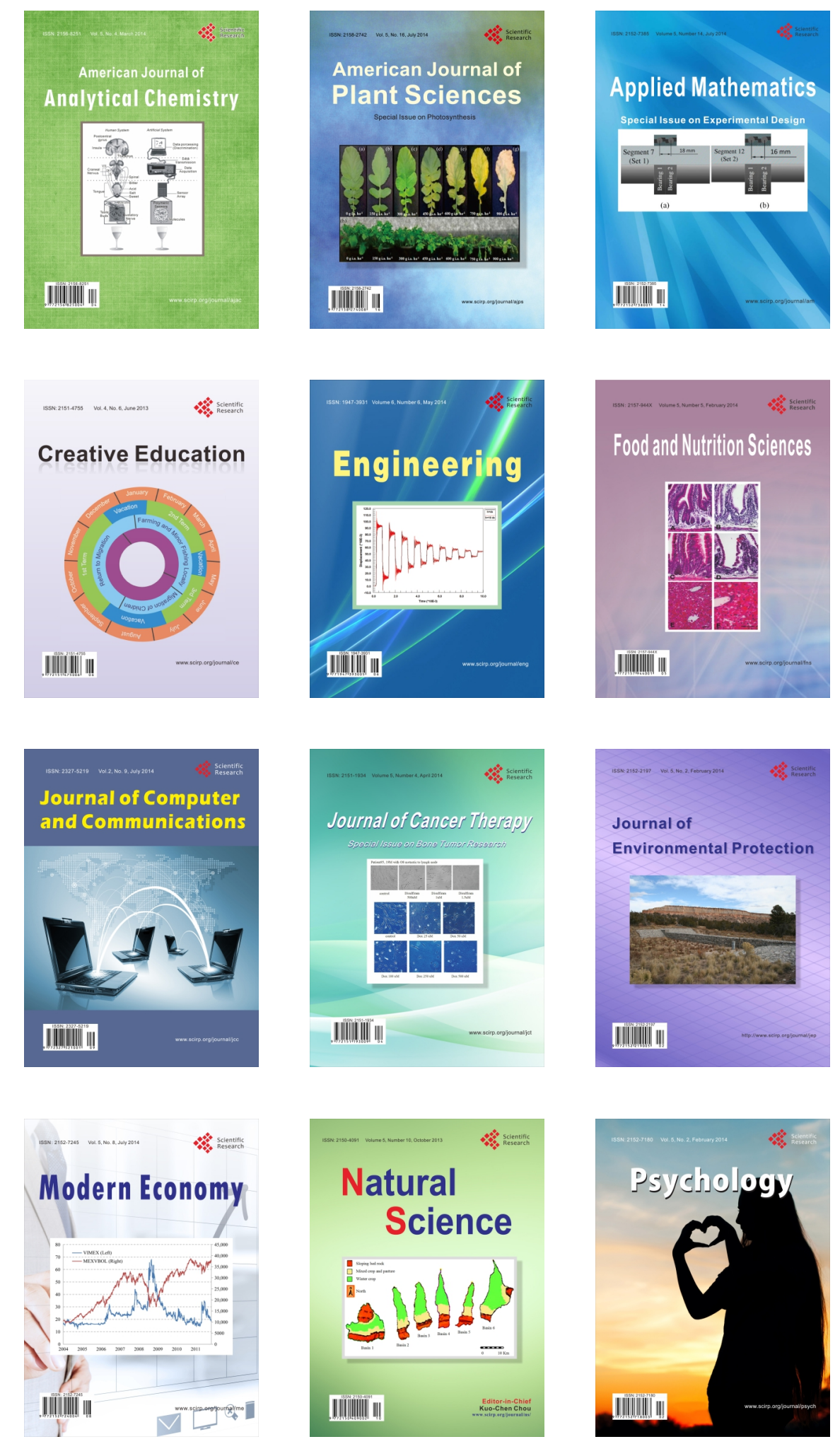